\title{
Signs of right ventricular myocyte hypertrophy in arrhythmogenic cardiomyopathy
}

\author{
Stefan Peters* \\ St.Elisabeth Hospital Salzgitter, Germany
}

\begin{abstract}
In human and mouse models mutations of desmoglein-2 produce a form of arrhythmogenic cardiomyopathy with massive necrosis and right ventricular myocyte hypertrophy.

In a cohort of 434 patients with typical arrhythmogenic cardiomyopathy it was searched for signs of right ventricular hypertrophy in standard ECG.

In 12 cases manifest or subtle signs of right ventricular hypertrophy were present (3\%) beyond minor or major ECG criteria of arrhythmogenic cardiomyopathy.

Although desmoglein-2 mutations have a frequency of $5-15 \%$ in arrhythmogenic cardiomyopathy additional signs of right ventricular hypertrophy are a hint of massive necrosis in arrhythmogenic cardiomyopathy thus producing right ventricular myocyte hypertrophy.
\end{abstract}

In an mouse model of desmoglein-2 mutated arrhythmogenic cardiomyopathy [1] myocyte right ventricular hypertrophy could be proven after damage and necrosis in an advanced form of arrhythmogenic cardiomyopathy.

Signs of right ventricular hypertrophy are not included in major and minor ECG criteria of arrhythmogenic cardiomyopathy [2].

The question is are there obvious signs of right ventricular hypertrophy in the ECG of a large amount of patients with typical arrhythmogenic cardiomyopathy.

Shallow $\mathrm{S}$ wave in lead V1 with deep $\mathrm{S}$ wave in lead V2 was described as marker of mild right ventricular hypertrophy $[3,4]$ as described in the ECG's of patients with mitral valve stenosis in combination with tricuspid insufficiency.

\section{Method}

The ECG's of 434 patients with typical arrhythmogenic cardiomyopathy ( 296 males, mean age $46.6 \pm 11.3$ years) were analyzed with regard to Sokolow-Lyon amplitude criteria, ST-T criteria, axis criteria and QRS criteria and shallow $S$ wave as mentioned above.

\section{Results}

In search for ECG signs of right ventricular hypertrophy there was one patient with Sokolow-Lyon criteria of right ventricular hypertrophy with an $\mathrm{R}$ wave in lead $\mathrm{V} 1>1.5 \mathrm{~mm}$ with right bundle branch block criteria (Figure 1).

Shallow S wave signs in lead V1 and V2 were present in 11 cases with typical arrhythmogenic cardiomyopathy (Figure 2).

\section{Conclusions}

What is interesting is the fact that obvious ECG signs of right ventricular hypertrophy are extremely rare in typical forms of arrhythmogenic cardiomyopathy with an event are of only $3 \%$.

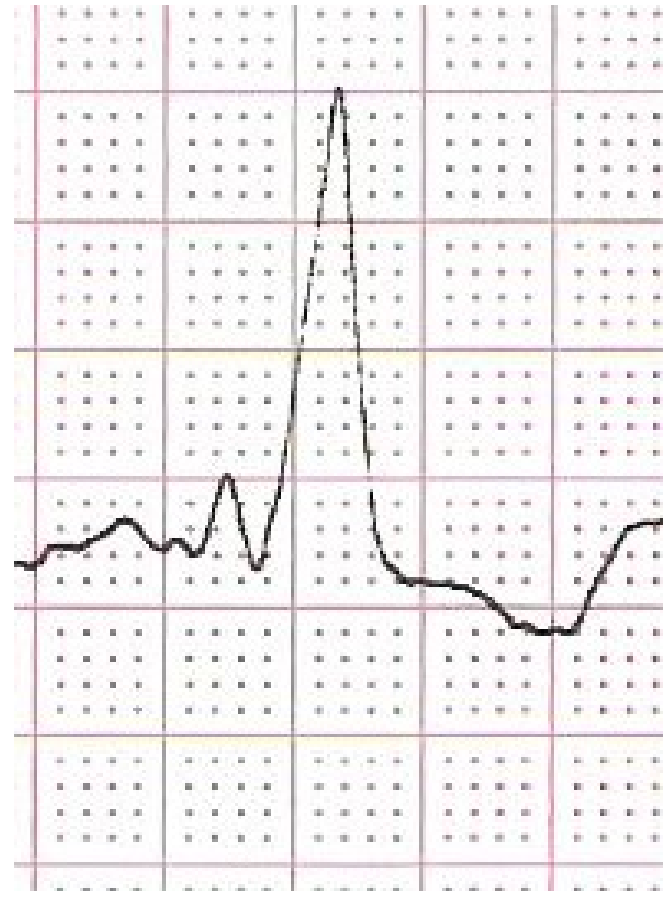

Figure 1. A case with advanced form of arrhythmogenic cardiomyopathy. In the ECG complete right bundle branch block and hints for right ventricular hypertrophy

${ }^{*}$ Correspondence to: Stefan Peters, Cardiology, St.Elisabeth Hospital Salzgitter, Liebenhaller Str. 20, 38259 Salzgitter, Germany, Tel: +39 5341824 679; E-Mail: H.u.S.Peters@t-online.de

Keywords: Arrhythmogenic cardiomyopathy, desmoglein-2, right ventricular hypertrophy

Received: March 20, 2019; Accepted: March 28, 2019; Published: March 29 , 2019 


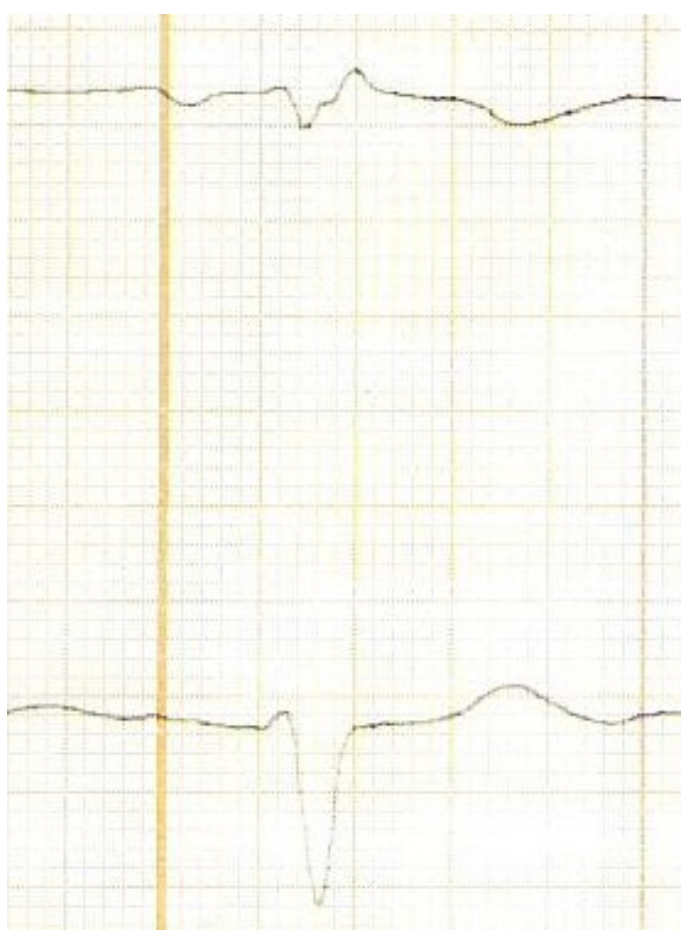

Figure 2. Typical example of shallow $\mathrm{S}$ wave in lead V1 and deep S wave in lead V2

Right ventricular myocyte hypertrophy is described in an mouse model with mutated desmoglein-2 [1]. After damage and necrosis of right ventricular myocytes right ventricular hypertrophy in most advanced forms of arrhythmogenic cardiomyopathy with left ventricular involvement.
Necrosis is not only described in animal models but also in an explanted heart of a proband carrying the DSG2-N266S mutation with massive calcification [5].

The rate of desmoglein-2 mutations in arrhythmogenic cardiomyopathy is about $10-15 \%$, but in the present cohort possibly only $3 \%$. The theory is that signs of right ventricular hypertrophy are desmoglein-2 mutation - dependent. Necrosis with massive calcification is only described in desmoglein-2 mutations in advanced forms of arrhythmogenic cardiomyopathy.

Special ECG features of arrhythmogenic cardiomyopathy are described for founder mutations like TMEM43 mutations and phospholamban mutations [6] and possibly right ventricular hypertrophy in desmoglein-2 mutations.

\section{References}

1. Gercek M, Gercek M, Kant S, Simsekyilmaz S, Kassner A, et al. (2017) Cardiomyocyte hypertrophy in arrhythmogenic cardiomyopathy. Am J Pathol 187: 752-766.

2. Marcus FI, McKenna WJ, Sherrill D, Basso C, Bauce B, et al. (2010) Diagnosis of arrhythmogenic right ventricular cardiomyopathy/dysplasia: proposed modification of the Task Force Criteria. Eur Heart J 31: 806-814.

3. Flowers NC, Horan LG (1972) Hypertrophy and infraction: Subtle signs of righ ventricular enlargement and their relative importance. In: Schlant RC, Hurst JW (Eds): Advances in Electrocardiography. New York: Grund \& Stratton.

4. Shubin H, Levinson DC (1958) The deep S wave in leads V1, V2, and V3 in right ventricular hypertrophy. Circulation 18: 410-417. [Crossref]

5. Pilichou K, Remme CA, Basso C, Campian ME, Rizzo S, Barnett P, et al. (2009) Myocyte necrosis underlies progressive myocardial dystrophy in mouse dsg2-related arrhythmogenic right ventricular cardiomyopathy. J Exp Med 206: 1787-1802.

6. Peters S (2018) Electrocardiographic criteria in founder mutations related to arrhythmogenic cardiomyopathy. J Cardiol Cardiovasc Med 3: 005-007

Copyright: (C2019 Peters S. This is an open-access article distributed under the terms of the Creative Commons Attribution License, which permits unrestricted use, distribution, and reproduction in any medium, provided the original author and source are credited. 